\title{
High-Density FIB-SEM Tomography Via Real-Time Imaging
}

\author{
E. L. Principe*, P.E. Sobol** \\ *Carl Zeiss SMT, Inc. Nanotechnology Systems Division \\ 555 Twin Dolphin Drive, Suite 130, Redwood City California, 94065 \\ **Monona Analytical, 6005 Midwood Ave, Monona Wisconsin, 53716 \\ Email: principe@smt.zeiss.com
}

The value of the ability to acquire, interrogate and display n-dimensional data sets has been well-established through various scientific disciplines. The biomedical sciences, in particular, have developed sophisticated methods to visualize volumetric image data. However, 3D tomographic data acquisition and volume visualization through the application of serial FIB sectioning has only just begun to emerge as a demonstrable method $^{1}$, with work completed by a small number of researchers. The FIB-SEM and FIB-Auger tomographic methods have demonstrated the ability to provide volumetric data resolution down to $10 \mathrm{~nm}$ or less ${ }^{1}$, and thus hold tremendous future potential for both material science and biomedical investigations. Yet even the best examples of what have been accomplished thus far in the field of FIB-based nanotomography reflect the fact that this technique is still in the early stages of its development.

Factors that limit wider utilization of FIB-based tomographic methods include the ease, speed and density of raw data collection. Another obstacle is implementing robust, yet versatile data analysis and volume visualization methods suitable for electron imaging. In principle the hardware has existed for over a decade to collect volumetric image data from a set of sequential FIB serial sections. Yet FIB-based nanotomography has, until now, remained a less practical application due to the time, effort and specialized data reduction expertise involved.

This paper will demonstrate that with the advent of high-resolution simultaneous SE imaging during the FIB sectioning process; it is now practical to acquire several hundred SEM image frames in the span of less than one hour in an automated fashion. It will be demonstrated that live imaging coupled with automated image recording facilitates the data acquisition process significantly, while easily providing lateral resolution at the nanoscale. In addition the high collection density translates to similar resolution along the direction normal to the cutting plane over a depth of several microns and greater.

The live data acquisition method is coupled to a data reduction process that allows convenient display of the high quality volume reconstructions through animated section sequences, exploration of sub-volumes and application of selective transparency. The figures on the following page show select examples. Aspects of data acquisition, data processing and approaches to quantification will be described.

\footnotetext{
${ }^{1}$ See for example Chapter 14 (Robert Hull) and Chapter 15 (E.L. Principe) and references contained therein, Introduction to Focused Ion Beams: Instrumentation, Theory, Techniques and Practice, Giannuzzi, Lucille A.; Stevie, Fred A. (Eds.). Springer-Verlag (2004).
} 

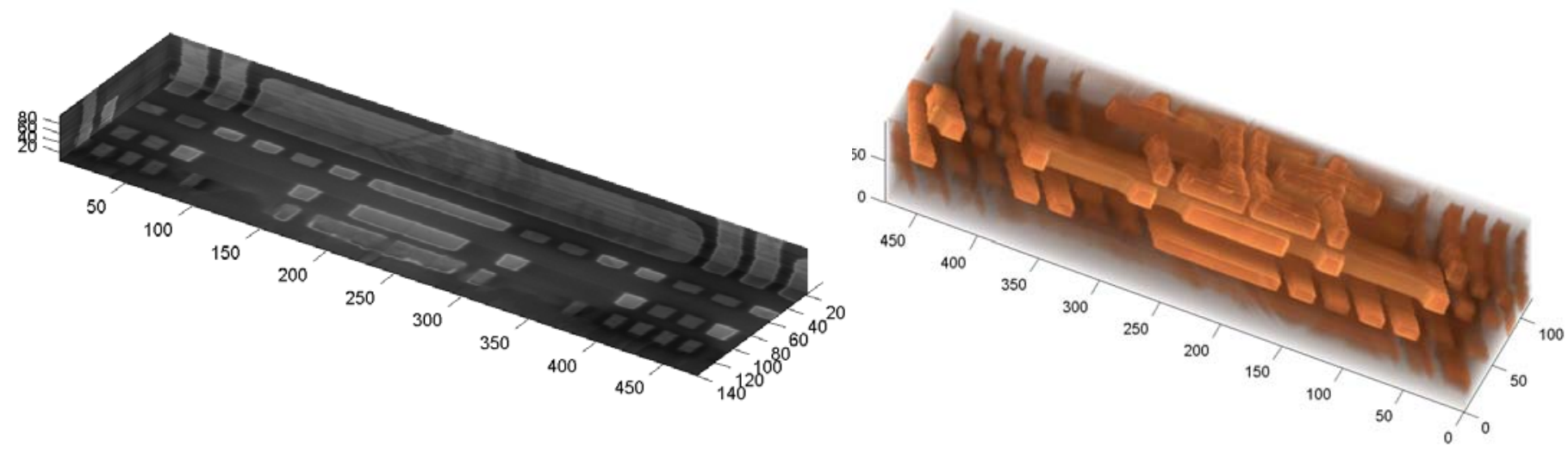

Figure 1. Multi-layer semiconductor structure. A cross-section cut-away view is shown at left. Note the detail in the copper grain structure on the top surface, which is perpendicular to the sectioning plane (front-to-back in image). At right is shown part of the same structure with selective transparency applied to the copper traces. Units are pixels. All representations can be animated to show rotating views from any angle, as well as animated sequences through any desired series of cutting planes. Sample courtesy of Eric Lifshin, SUNY at Albany Nanotechnology Center, College of Nanoscale Science \& Engineering.
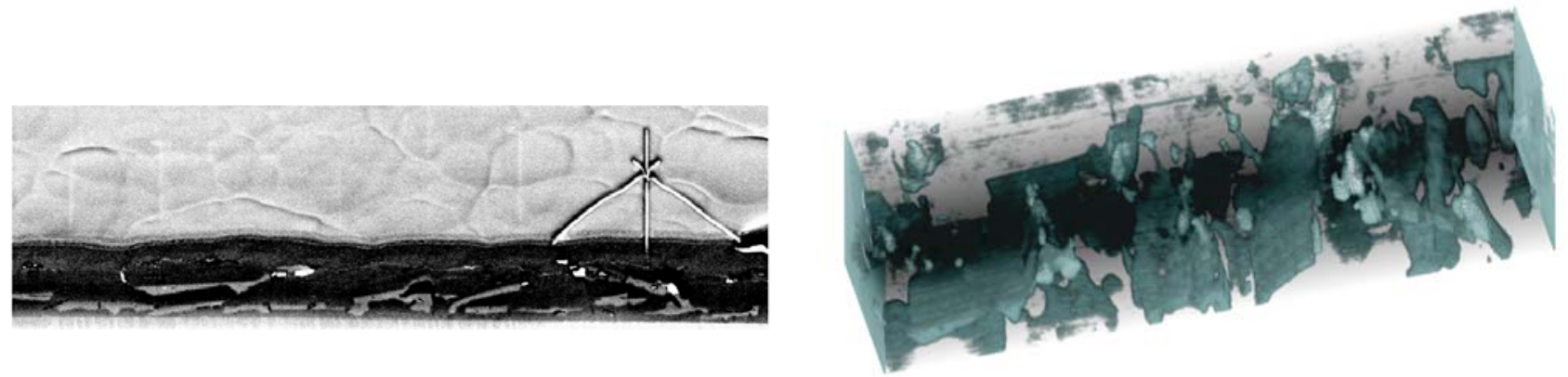

Figure 2. High-Tc superconductor thin film with second phases (bright areas in crosssection). At left is one frame of the original movie sequence. At right, a volumetric reconstruction with selective transparency applied to highlight the second phase material. Geometric complexities are revealed of the original structures appearing in the 2D crosssection on the left. Sample courtesy of Dean Miller, Argonne National Laboratory.

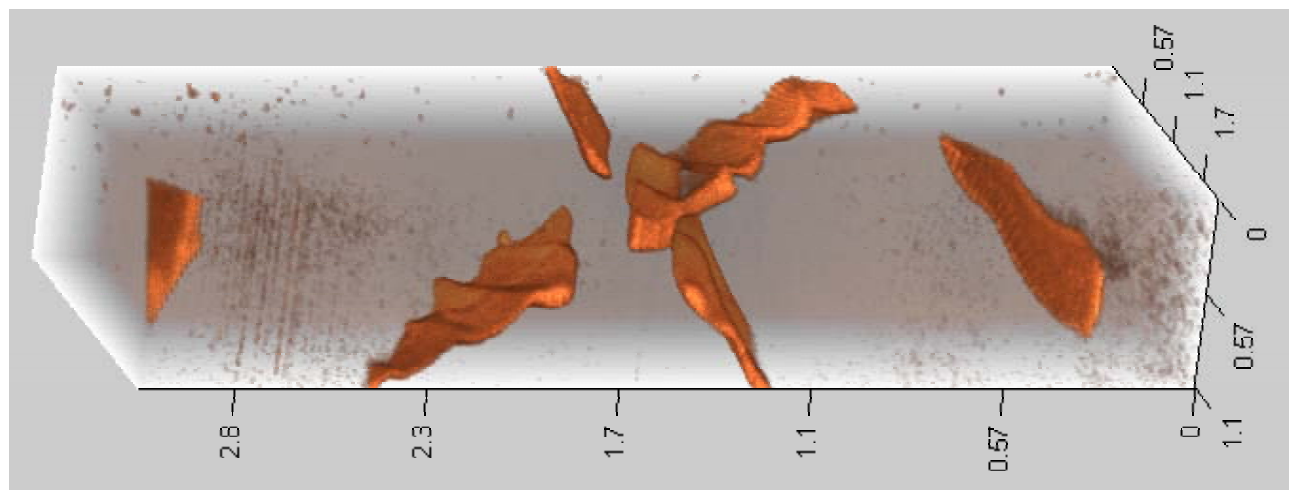

Figure 3. Selective transparency of a phase detail formed in heat affected zone of a weld. Axes units are microns. Sample courtesy of Mahesh Chaturvedi, University of Manitoba. 